\title{
Surface Phase Metastability during Langmuir Evaporation
}

\author{
K. Hannikainen, D. Gomez, J. Pereiro®, Y. R. Niu, and D. E. Jesson॰ \\ School of Physics and Astronomy, Cardiff University, Cardiff CF24 3AA, United Kingdom
}

(Received 23 April 2019; published 1 November 2019)

\begin{abstract}
We have directly imaged the spontaneous formation of metastable surface phase domains on GaAs(001) during Langmuir evaporation. Eventually, these metastable phases transform to the thermodynamically stable parent phase, producing a dynamic phase coexistence with a temperature dependent, time-averaged coverage. Monte Carlo simulations are used to identify the key kinetic processes and investigate the interplay between phase metastability and evolving surface morphology. This is used to explain the measured temperature dependence of the time-averaged coverage.
\end{abstract}

DOI: 10.1103/PhysRevLett.123.186102

Free evaporation of atoms from a surface into a vacuum (Langmuir evaporation) has been extensively studied over the years [1-6]. In addition to being of fundamental scientific importance, it is directly relevant to the processing and growth of thin films and nanostructures across a wide range of technologies [1-11]. Here, we apply low energy electron microscopy (LEEM) to the technologically important $\mathrm{GaAs}(001)$ surface and reveal a striking new feature of Langmuir evaporation. Metastable surface phases are seen to form spontaneously, as unstable subsurface layers are exposed by evaporation. Eventually, these phases convert to the thermodynamically stable parent phase, producing a temperature-dependent, time-averaged metastable-phase coverage. This dynamic phase coexistence has important practical implications for the application of phase diagrams to interpret evaporating surfaces under vacuum, where a single phase is usually assumed to be present. We believe such phase metastability, induced by evaporation, is likely to be relevant for the optimization of thin film growth conditions across a wide range of material systems.

Experiments were performed under ultrahigh vacuum, in a LEEM modified for III-V MBE [12]. The temperature was calibrated by observing Ga droplet generation [4] and various surface-phase transformations [13]. An undoped GaAs(001) sample was degassed at $300^{\circ} \mathrm{C}$ for $24 \mathrm{~h}$. This was followed by annealing at $580^{\circ} \mathrm{C}$ for $2 \mathrm{~h}$ to remove the surface oxide. The sample temperature was then increased above the congruent evaporation temperature to $650{ }^{\circ} \mathrm{C}$ to create $\mathrm{Ga}$ droplets of radius $\sim 2 \mu \mathrm{m}$ which were allowed to run across the surface [14,15]. This created smooth planar (001) regions which we utilize for our imaging experiments [16]. Finally, the Ga droplets were completely removed by annealing below the congruent evaporation temperature at $570{ }^{\circ} \mathrm{C}$.

The sample was then heated above $580^{\circ} \mathrm{C}$ where we would expect to observe the well-studied $c(8 \times 2)$ reconstruction, which is widely accepted to be stable under these conditions [13,17-19]. Figure 1 contains snapshots taken from a LEEM movie [20] of $\operatorname{GaAs}(001)$ at $598^{\circ} \mathrm{C}$, obtained under bright-field imaging conditions at an incident electron energy of $8.6 \mathrm{eV}$. All images have been smoothed utilizing standard interpolation methods. At time $t=0$, panel (a) displays uniform, bright intensity corresponding to an expected $c(8 \times 2)$ surface. Two steps, which are receding due to evaporation, are also indicated by the arrows. These are of bilayer height, separating $c(8 \times 2)$ reconstructed terraces. Surprisingly, after 384 s, a dark patch nucleates in the center of the terrace [panel (b)] and grows [panels (c) and (d)]. By careful dark-field imaging $[20,21]$, we are able to confirm that the dark contrast corresponds to the $(6 \times 6)$ reconstruction. The $(6 \times 6)$ patch continues to grow until $t=1416 \mathrm{~s}$ when a small region of $c(8 \times 2)$ phase nucleates within the $(6 \times 6)$ patch (a) $t=0 s$

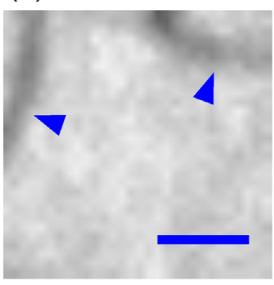

(d) $\mathrm{t}=1408 \mathrm{~s}$

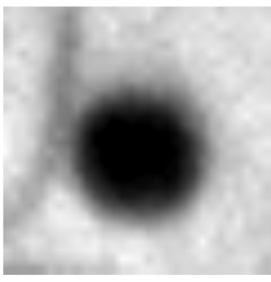

(b) $t=384 \mathrm{~s}$

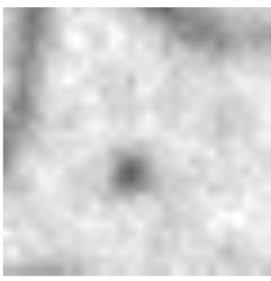

(e) $\mathrm{t}=1416 \mathrm{~s}$

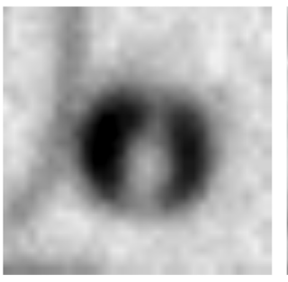

(c) $\mathrm{t}=1024 \mathrm{~s}$

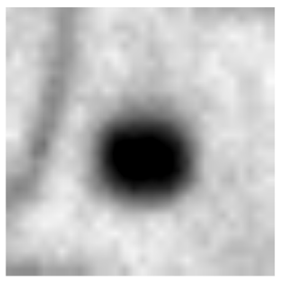

(f) $\mathrm{t}=1448 \mathrm{~s}$

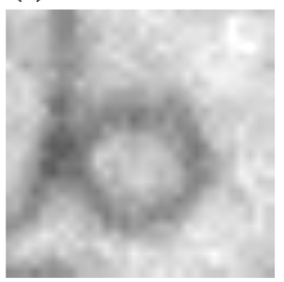

FIG. 1. Snapshots taken from a LEEM movie [20] revealing the fundamental mechanism of surface-phase metastability. The $c(8 \times 2)$ phase appears bright, while $(6 \times 6)$ and steps [indicated with arrows in (a)] appear dark. The scale bar in (a) is $0.1 \mu \mathrm{m}$ and the sample temperature is $598^{\circ} \mathrm{C}$. 
[panel (e)]. The $c(8 \times 2)$ region then grows at the expense of the $(6 \times 6)$ phase until it is completely consumed, and only $c(8 \times 2)$ remains bounded by a bilayer height step loop. The nucleation and growth of $(6 \times 6)$ patches, followed by subsequent annihilation by $c(8 \times 2)$ occurs as discrete events across the entire surface. This dynamics produces a time-averaged phase coexistence between $(6 \times 6)$ and $c(8 \times 2)$ domains.

To explain the appearance of the $(6 \times 6)$ patches in Fig. 1, we must consider the mechanisms of Langmuir evaporation. The nucleation and growth of surface macrovacancies (Lochkeime) is known to play an important role in the evaporation of surfaces [22-24]. Here, surface vacancies typically form stable monolayer height clusters in a terrace, and the resulting step loop expands as atoms evaporate from the surface, causing the macrovacancy to grow. This mechanism would seem to be consistent with our observations in Fig. 1, but with one important exception. The macrovacancy nucleation and growth in panel (b) is associated with a $(6 \times 6)$ reconstruction rather than

(a)

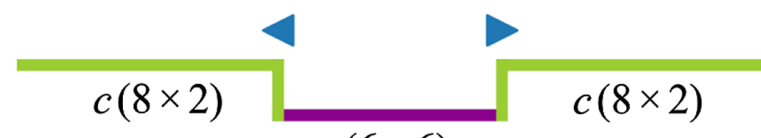

(b)

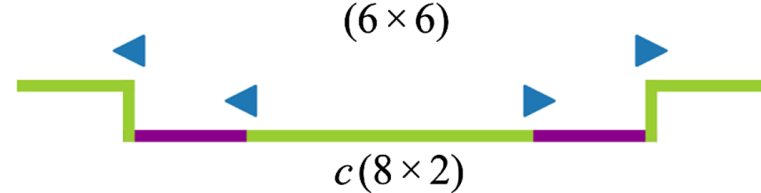

(c)

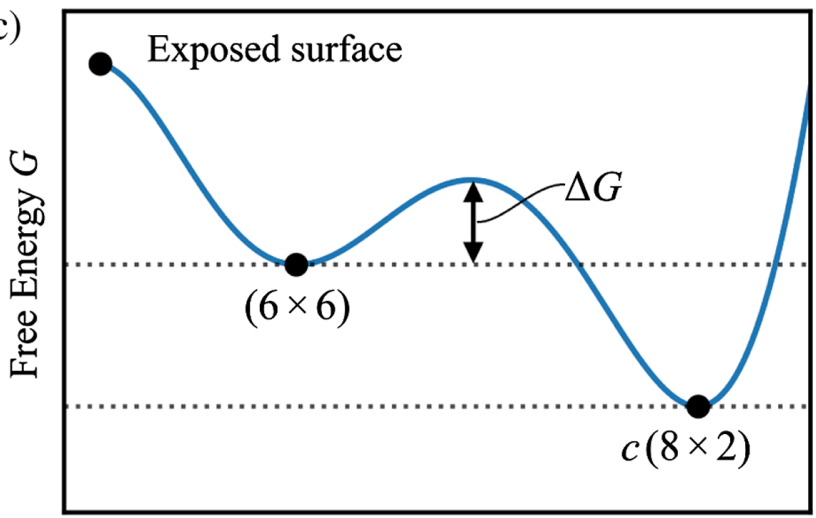

Reaction Coordinate

FIG. 2. Cross-sectional schematic of Lochkeim formation and surface-phase metastability. (a) A Lochkeim formation forms in the $c(8 \times 2)$ phase (green line) and bilayer height steps propagate as atoms evaporate into the vacuum. The freshly exposed surface is the $(6 \times 6)$ phase (purple line). (b) $c(8 \times 2)$ nucleates within the $(6 \times 6)$ phase and the phase boundaries propagate until only $c(8 \times 2)$ is present. (c) Schematic representation of the change in surface free energy (per unit area) of the exposed surface. The exposed surface is unstable and transforms into the metastable $(6 \times 6)$ phase. An activation energy barrier $\Delta G$ exists for the conversion of the metastable $(6 \times 6)$ to the thermodynamically stable $c(8 \times 2)$. the stable $c(8 \times 2)$. This is illustrated schematically in Fig. 2(a). To explain this, we note that the freshly exposed surface during macrovacancy nucleation is in an unstable state and does not necessarily have to transform directly into the most thermodynamically stable state. Rather, it can transform into a metastable intermediate state as conjectured by Ostwald [25-28] [see Fig. 2(c)]. It seems likely that the route to $(6 \times 6)$ from the freshly exposed, unstable surface will be influenced by surface strain generated by the initial small step loop. However, the atomic-scale details of the initial nucleation process are below our instrumental resolution. Eventually, the metastable $(6 \times 6)$ phase converts to the stable $c(8 \times 2)$ phase via the nucleation of $c(8 \times 2)$ regions within the $(6 \times 6)$ phase [panels $1(\mathrm{e})$, 2(b), and 2(c)]. The $c(8 \times 2)$ phase then rapidly grows, leaving behind a bilayer height step loop on pure $c(8 \times 2)$ [panel 1(f)]. Hence, this dynamics of nucleation, growth, and annihilation gives rise to a time-averaged coverage of $(6 \times 6)$.

To quantify this dynamic mechanism we have measured the time-averaged coverage of $(6 \times 6)$ as a function of temperature $T$, as displayed in Fig. 3. The data were averaged over progressively shorter times and surface areas with increasing $T$ due to the faster evaporation kinetics at higher temperatures. This ranged from $3 \mathrm{~h} / 15 \mu \mathrm{m}^{2}$ at the lowest $T\left(581^{\circ} \mathrm{C}\right)$ to $30 \mathrm{~min} / 3 \mu \mathrm{m}^{2}$ at the highest $\left(639^{\circ} \mathrm{C}\right)$. Below $580^{\circ} \mathrm{C}$, the kinetics of evaporation became too slow to obtain time-averaged data. It can be seen that the $(6 \times 6)$ coverage decreases from $\sim 9 \%$ at $580{ }^{\circ} \mathrm{C}$ to around $0.1 \%$ at $640{ }^{\circ} \mathrm{C}$. The existence of $(6 \times 6)$ in this

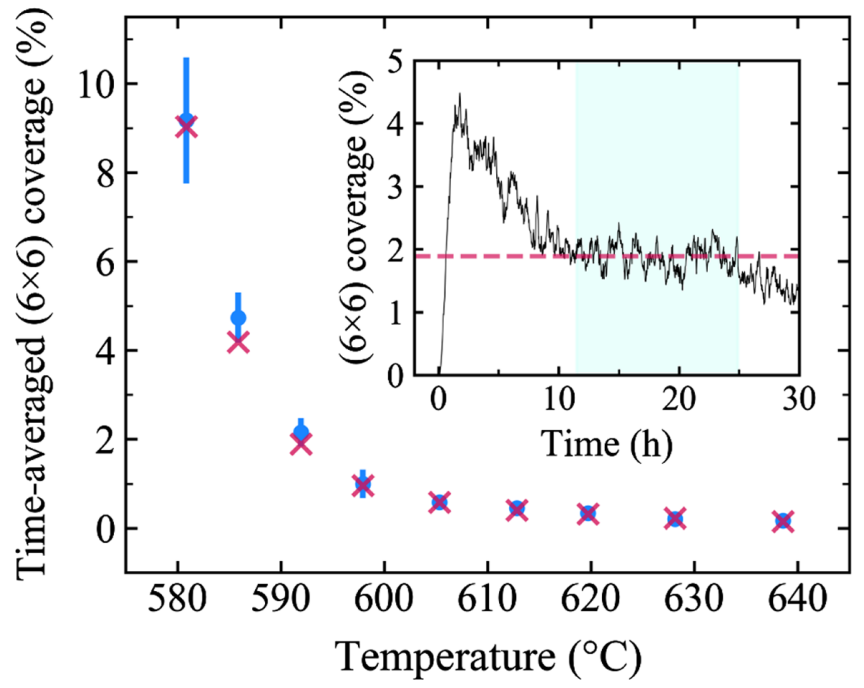

FIG. 3. Time-averaged $(6 \times 6)$ coverage as a function of temperature. The circles are experimental values and the crosses were calculated from the MC simulations. The inset shows the time evolution of the $(6 \times 6)$ coverage produced by the MC simulation at $592^{\circ} \mathrm{C}$. The dashed line shows the time-averaged coverage obtained from the shaded region (see text). Error bars are computed as standard deviations from the mean. 
temperature range is surprising as it is generally assumed that only one phase, $c(8 \times 2)$, is present. It is likely that the transient nature and relatively small time-averaged $(6 \times 6)$ coverage explains why this surface-phase metastability has not been observed previously.

To explain the temperature dependence of the coverage we have developed a Monte Carlo (MC) simulation model which is governed by a set of simple rules derived from our LEEM movies. We consider a $10 \mu \mathrm{m}$ square portion of a $\operatorname{GaAs}(001)$ surface, held at temperature $T$, subject to periodic boundary conditions. Pointlike Lochkeime are allowed to form at a uniform rate of $J_{w}$ per unit area. The macrovacancy step loop associated with the Lochkeim is then expanded at a uniform velocity $v$, as GaAs evaporates, revealing a bilayer deep, circular $(6 \times 6)$ terrace. This mimics Figs. 1(a)-1(d) and the cross-sectional schematic in Fig. 2(a). The nucleation of $c(8 \times 2)$ on this terrace [Figs. 1(e) and 2(b)] is taken to occur at a rate $\rho$ per unit area. Since our movies show that the transformation of $(6 \times 6)$ to $c(8 \times 2)$ is fast on the timescale of all other evaporation-related kinetic processes, including Lochkeim formation and $(6 \times 6)$ terrace growth, we allow an instantaneous conversion of $(6 \times 6)$ to $c(8 \times 2)$ across the entire $(6 \times 6)$ terrace.

These simple rules adequately describe the fundamental process of $(6 \times 6)$-phase metastability shown in Fig. 1 . However, to obtain a full, quantitative agreement with the experimentally observed coverage, our LEEM movies indicate that we must also incorporate several secondary processes into the MC model.

(i) We observe that Lochkeime form more readily on $(6 \times 6)$ than on $c(8 \times 2)$ (see Sec. II. A of [20]). We therefore introduce an additional rate $J_{b}$ for the rate of Lochkeim formation per unit area of $(6 \times 6)$. In accordance with observation, $J_{b}$ is taken as uniform across a $(6 \times 6)$ domain but only up to one Lochkeim is allowed to form per $(6 \times 6)$ terrace. In addition, we observe that when this mechanism results in an "inverted wedding cake" of $(6 \times 6)$ terraces, it is always the outer (highest) terrace which first transforms to $c(8 \times 2)$ (propagation of the stable phase stops at the step). This may reflect some stabilization of the inner $(6 \times 6)$ domains by surface stress.

(ii) When a $c(8 \times 2)$ terrace attains a critical radius $R_{c}$, one or more Lochkeime form at the center of the terrace (see Sec. II. B of [20]). We note that an analogous phenomenon has been observed during the epitaxial growth of $\mathrm{Ag}$ [29]. For simplicity, we assume only one Lochkeim nucleation event per critically sized $c(8 \times 2)$ terrace. This mechanism serves to maintain inverted wedding cake structures when $(6 \times 6)$ domains transform to $c(8 \times 2)$ before undergoing Lochkeim nucleation.

(iii) $(6 \times 6)$ domains may coalesce such that a single nucleation event at rate $\rho$ transforms the entire terrace to $c(8 \times 2)$ (see Sec. II. C of [20]).

(iv) When a $(6 \times 6)$ domain coalesces with a $c(8 \times 2)$ terrace, it rapidly transforms to $c(8 \times 2)$ (see Sec. II. D of
[20]). This conversion to $c(8 \times 2)$ is incorporated in the model as an instantaneous event.

$J_{w}, J_{b}, \rho$, and $v$ are measured directly from the LEEM movies at nine different temperatures in the $580-640{ }^{\circ} \mathrm{C}$ range (Fig. 4). To a good approximation, the step velocity is independent of the nature of the phases either side of the step. So only one velocity is used for all steps at a given $T$. Step bunching can affect the velocity, but this has a negligible effect on the simulations and so is neglected. $J_{b}$ and $\rho$ are determined from the measured distribution of $(6 \times 6)$ terrace size during respective Lochkeim and $c(8 \times 2)$ nucleation [20]. The measured value of $R_{c}=$ $0.17 \mu \mathrm{m}$ is found to be approximately constant over the temperature range of interest.

We fit the $T$ dependence of $J_{w}, J_{b}, \rho$, and $v$ to the standard Arrhenius form, $x=x_{0} \exp \left(-E_{a} / k T\right)$ where $x_{0}$ and $E_{a}$ are the respective prefactor and energy barrier with $k$ equal to Boltzmann's constant (see Fig. 4). The determined prefactors and energy barriers contained in the Fig. 4 caption are used in the MC simulations. However, these values should not be interpreted physically since it is well appreciated that non-Arrhenius temperature dependence can arise during the complex kinetic processes associated with evaporation [24]. Rather, the Arrhenius form used here should be viewed as a convenient fit to the measured data.

MC simulations were run for the nine temperatures corresponding to the LEEM measurements (see the MC simulation movie [20]). They begin with a pure $c(8 \times 2)$

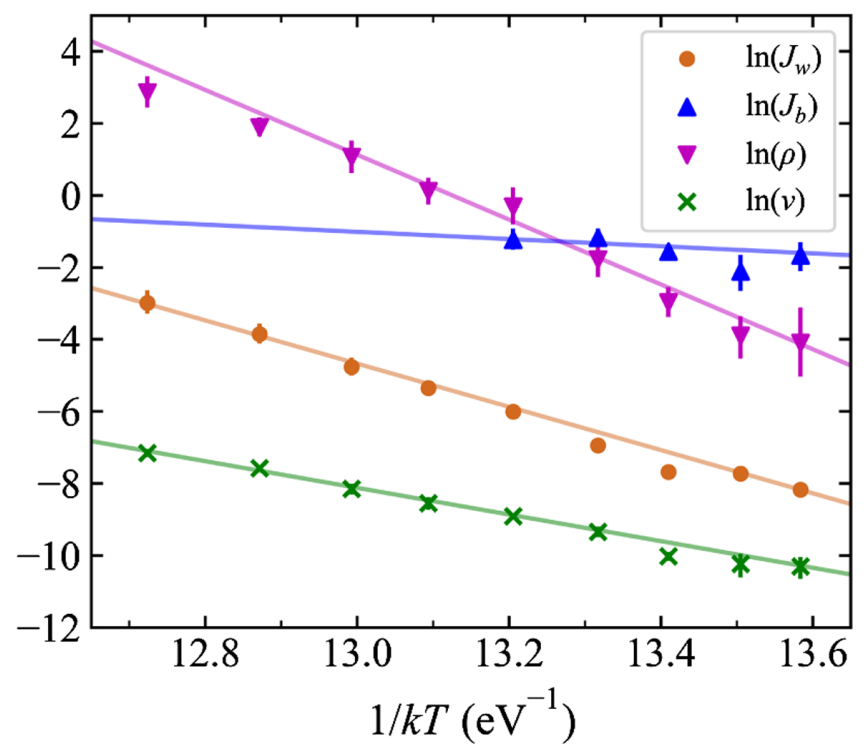

FIG. 4. Measured rates of the key kinetic processes used in the MC simulations as a function of $T . \ln \left(J_{w}\right), \ln \left(J_{b}\right), \ln (\rho)$, and $\ln (v)$ are plotted against $1 / k T$ with $J_{w}, J_{b}$, and $\rho$ in units of $\mu \mathrm{m}^{-2} \mathrm{~s}^{-1}$ and $v$ in $\mu \mathrm{m} \mathrm{s}^{-1}$. The respective prefactors of $J_{w}, J_{b}, \rho$, and $v$ obtained from the linear fits to the measured data are $7.0 \times 10^{31} \mu \mathrm{m}^{-2} \mathrm{~s}^{-1}, \quad 1.6 \times 10^{5} \mu \mathrm{m}^{-2} \mathrm{~s}^{-1}, 2.0 \times 10^{51} \mu \mathrm{m}^{-2} \mathrm{~s}^{-1}$, and $2.3 \times 10^{17} \mu \mathrm{m} \mathrm{s}^{-1}$ and the respective energy barriers are $6.0,1.0,9.0$, and $3.7 \mathrm{eV}$ (see text). 
surface exposed to the vacuum. As the simulations proceed, the $(6 \times 6)$ coverage initially increases before decaying and settling in to a steady state. The coverage and surface morphology associated with this steady state are used to compare with experiment. For the three lowest temperatures, rather than a strict steady state, we find a longperiod decrease in $(6 \times 6)$ as shown by the inset in Fig. 3 for $592^{\circ} \mathrm{C}$. However, this long decay is experimentally inaccessible and the shaded quasi-steady-state plateau region is used to determine the coverage for this, and the other two lowest temperature cases.

The time-averaged $(6 \times 6)$ coverage evaluated by MC simulations is compared with experiment in Fig. 3. Excellent agreement is found across the entire temperature range. Note that the fits in Fig. 4 were carefully tuned within the error bars to optimize agreement with experiment. To explain the decrease in $(6 \times 6)$ coverage with increasing $T$ we compare snapshots of the experimental and simulated surface morphology at low, intermediate, and high temperatures in Fig. 5. Again, the general reproduction of the salient experimental features by the simulations is very good. Both experiment and simulation indicate that there is a clear change in surface morphology intrinsically linked to the varying $(6 \times 6)$ coverage with $T$. By combining the MC simulation of surface morphology in Fig. 5 with the measured rates of the key kinetic processes in Fig. 4, we can now explain the link between evolving morphology and the $T$-dependent $(6 \times 6)$ coverage measurements in Fig. 3.

(a)

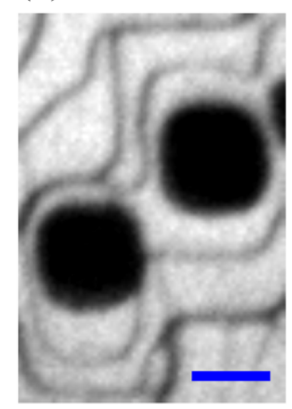

(d)

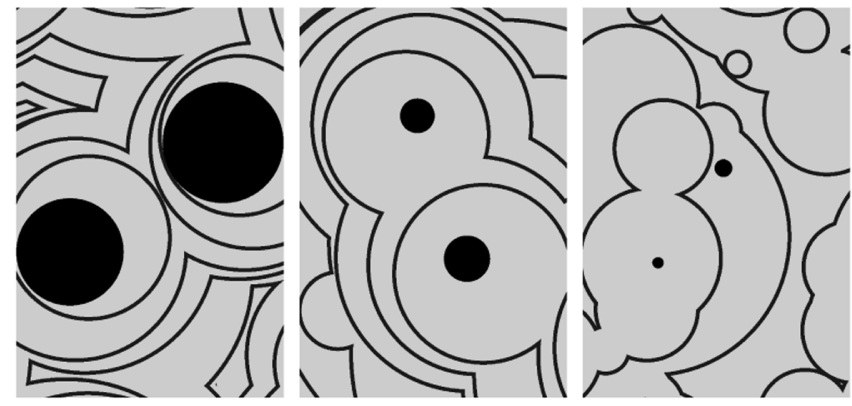

FIG. 5. Snapshots of evolving surface morphology taken from LEEM movies at (a) $581{ }^{\circ} \mathrm{C}$, (b) $598^{\circ} \mathrm{C}$, and (c) $639^{\circ} \mathrm{C}$ and $\mathrm{MC}$ simulation movies at (d) $581^{\circ} \mathrm{C}$, (e) $598^{\circ} \mathrm{C}$, and (f) $639^{\circ} \mathrm{C}$. Dark areas correspond to $(6 \times 6)$ terraces. The scale bar in (a) is $0.2 \mu \mathrm{m}$.
At low $T$, it can be seen from Fig. 4 that $J_{b}>\rho$. Lochkeim nucleation is therefore more likely to occur on metastable $(6 \times 6)$ domains before they transform to $c(8 \times 2)$. Furthermore, the relatively high $J_{b} / v$ ratio in this regime promotes Lochkeim nucleation on $(6 \times 6)$ domains when they are still relatively small. This gives rise to a surface populated with inverted wedding cakes resulting from multilayer evaporation, as shown in Figs. 5(a) and 5(d) (also see the low T MC simulation [20]). Note that the outer (upper) $(6 \times 6)$ terrace of an inverted wedding cake is of an annular shape and provides a limited surface area for $c(8 \times 2)$ nucleation. This tends to preserve these structures which are responsible for the high $(6 \times 6)$ coverage measured at low $T$ in Fig. 3.

With increasing $T$, the inverted wedding cake structures become less pronounced. $\rho$ increases faster than the step velocity $v$ (Fig. 4$)$ so that outer $(6 \times 6)$ domains transform to $c(8 \times 2)$ at smaller sizes [panels 5(b), 5(c), 5(e), and 5(f) and the intermediate $T$ MC simulation [20]]. This decreases the overall time-averaged $(6 \times 6)$ coverage with increasing $T$ (Fig. 3).

Eventually, $\rho$ surpasses $J_{b}$ at higher $T$ (Fig. 4) and so fewer $(6 \times 6)$ domains undergo a second Lochkeim nucleation event during their shorter lifetime. Furthermore, the large value of $J_{w}$ in this regime facilitates terrace coalescence. As discussed earlier, coalescence can also further decrease the overall $(6 \times 6)$ coverage via the mechanisms in Secs. II. C, II. D in Supplemental Material [20]. Evaporation therefore proceeds in a layer-by-layer mode at high $T$, where fewer atomic layers are exposed at the surface [see panels 5(c) and 5(f) and the high $T$ MC simulation [20] ]. The enhanced rate of $c(8 \times 2)$ nucleation and the absence of inverted wedding cake structures explains the low $(6 \times 6)$ coverage in this regime.

The observation of surface-phase metastability during Langmuir evaporation is surprising but the phenomenon should, in fact, occur quite widely. An unstable subsurface layer, suddenly exposed by evaporation, does not necessarily have to transform directly into the most thermodynamically stable state. Instead, the unstable surface can transform into a metastable intermediate state, as empirically described by Ostwald [25]. Figure 5 illustrates the intricate interplay between the kinetic processes of Langmuir evaporation and phase metastability which determines the time-averaged coverage as a function of $T$ (Fig. 3). Such phase coexistence is a result of kinetics, not thermodynamics, and is distinct from coexistence resulting from long-range electrostatic and elastic interactions between surface domains [30,31].

In summary, we have observed surface-phase metastability during Langmuir evaporation of $\mathrm{GaAs}(001)$. This gives rise to a dynamic-phase coexistence which has previously gone unnoticed, possibly due to a lack of real-time imaging of this surface. The direct observation of metastable phases provides new insights into the kinetic processes of Langmuir evaporation. Evaporation is 
dominated by inverted wedding cake structures at low $T$, creating a significant time-averaged metastable-phase coverage. With increasing $T$, such structures become less pronounced, with a concomitant decrease in $(6 \times 6)$ coverage as evaporation tends towards a layer-by-layer mode. Often, for purposes of growth, a single surface phase is required. Since surface-phase metastability is likely to occur across a wide range of materials systems, it might therefore have broad technological relevance for the growth and processing of thin films under vacuum.

Information on the data that underpins the results presented here, including how to access them, can be found in the Cardiff University data catalogue [32].

We are grateful to Jerry Tersoff for helpful discussions during the early stages of this project. Numerical computations were undertaken using the supercomputing facilities of Advanced Research Computing at Cardiff (ARCCA) on behalf of the HPC Wales and Supercomputing Wales (SCW) projects. The authors acknowledge support from EPSRC research Grants No. EP/P023452/1 and No. EP/ N022661/1. This project also has received funding from the European Union's Horizon 2020 research and innovation programme under the Marie Sklodowska-Curie Grant agreement No. 701246.

*Corresponding author. dej.workaddress@gmail.com

[1] C. T. Foxon, J. A. Harvey, and B. A. Joyce, J. Phys. Chem. Solids 34, 1693 (1973).

[2] C. Y. Lou and G. A. Somorjai, J. Chem. Phys. 55, 4554 (1971).

[3] B. Goldstein, D. J. Szostak, and V. S. Ban, Surf. Sci. 57, 733 (1976).

[4] J. Y. Tsao, Materials Fundamentals of Molecular Beam Epitaxy (Academic Press, San Diego, 1993).

[5] C. Chatillon and D. Chatain, J. Cryst. Growth 151, 91 (1995).

[6] J. Tersoff, D. E. Jesson, and W. X. Tang, Phys. Rev. Lett. 105, 035702 (2010).

[7] N. Isomura, S. Tsukamoto, K. Iizuka, and Y. Arakawa, J. Cryst. Growth 301-302, 26 (2007).

[8] D. Bimberg, M. Grundmann, and N. N. Ledentsov, Quantum Dot Heterostructures (John Wiley \& Sons, Chichester, 1999).

[9] P. Bhattacharya, S. Ghosh, and A. D. Stiff-Roberts, Annu. Rev. Mater. Res. 34, 1 (2004).
[10] B. A. Joyce and D. D. Vvedensky, Mater. Sci. Eng. R 46, 127 (2004).

[11] J. Orton and T. Foxon, Molecular Beam Epitaxy: A Short History (Oxford University Press, Oxford, 2015).

[12] D. E. Jesson and W.X. Tang, in Microscopy, edited by A. Mendez-Vilas and J. Diaz (Formatex, Badajoz, 2010).

[13] A. Ohtake, Surf. Sci. Rep. 63, 295 (2008).

[14] J. Tersoff, D. E. Jesson, and W. X. Tang, Science 324, 236 (2009).

[15] E. Hilner, A. A. Zakharov, K. Schulte, P. Kratzer, J. N. Andersen, E. Lundgren, and A. Mikkelsen, Nano Lett. 9, 2710 (2009).

[16] C. Zheng, W.-X. Tang, and D. E. Jesson, J. Vac. Sci.\& Technol. A 34, 043201 (2016).

[17] C. X. Zheng, J. Tersoff, W. X. Tang, A. Morreau, and D. E. Jesson, Phys. Rev. B 93, 195314 (2016).

[18] A. Ohtake, S. Tsukamoto, M. Pristovsek, N. Koguchi, and M. Ozeki, Phys. Rev. B 65, 233311 (2002).

[19] M. Pristovsek, S. Tsukamoto, A. Ohtake, N. Koguchi, B. G. Orr, W. G. Schmidt, and J. Bernholc, Phys. Status Solidi (b) 240, 91 (2003).

[20] See Supplemental Material at http://link.aps.org/ supplemental/10.1103/PhysRevLett.123.186102 for a LEEM movie of $\mathrm{GaAs}(001)$ Langmuir evaporation, for LEEM dark-field imaging of the $(6 \times 6)$ surface phase, for a discussion of additional processes and determining $J_{b}$ and $\rho$ from the LEEM movies, and for a Monte Carlo simulation movie of surface-phase metastability during Langmuir evaporation.

[21] Y. R. Niu, J. Pereiro, D. Gomez, and D. E. Jesson, Ultramicroscopy 200, 79 (2019).

[22] J. J. Metois and D. E. Wolf, Surf. Sci. 298, 71 (1993).

[23] M. Mundschau, E. Bauer, W. Telieps, and W. Swieh, Surf. Sci. 223, 413 (1989).

[24] A. Pimpinelli and J.-J. Métois, Phys. Rev. Lett. 72, 3566 (1994).

[25] W. Ostwald, Z. Phys. Chem. 22, 289 (1897).

[26] S.-Y. Chung, Y.-M. Kim, J.-G. Kim, and Y.-J. Kim, Nat. Phys. 5, 68 (2009).

[27] L. O. Hedges and S. Whitelam, J. Chem. Phys. 135, 164902 (2011).

[28] I. N. Stranski and D. Totomanow, Z. Phys. Chem. 163A, 399 (1933).

[29] J. Tersoff, A. W. D. van der Gon, and R. M. Tromp, Phys. Rev. Lett. 72, 266 (1994).

[30] D. Vanderbilt, Surf. Sci. 268, L300 (1992).

[31] J. B. Hannon, F.-J. zu Heringdorf, J. Tersoff, and R. M. Tromp, Phys. Rev. Lett. 86, 4871 (2001).

[32] K. Hannikainen, D. Gomez, J. Pereiro, Y. R. Niu, and D. E. Jesson, Cardiff University data catalogue, https://doi.org/ 10.17035/d.2019.0085857240, 2019. 